\title{
Molecular diagnosis of spinal and bulbar muscular atrophy in Slovakia
}

\author{
Zelinkova $\mathrm{H}^{1}$, Lexova Kolejakova $\mathrm{K}^{1}$, Spalek $\mathrm{P}^{2}$, Chandoga $\mathrm{J}^{1}$, Konkolova $\mathrm{J}^{1}$, Bohmer $\mathrm{D}^{1}$ \\ Institute of Medical Biology, Genetics and Clinical Genetics, Comenius University Faculty of Medicine and University Hospital in \\ Bratislava, Department of Molecular and Biochemical Genetics, Bratislava, Slovakia. hana.zelinkova@gmail.com
}

\section{ABSTRACT}

OBJECTIVES: Molecular-genetic analysis is a determining step in setting the diagnosis of spinal and bulbar muscular atrophy (SBMA). We present the first nation-wide study and experience with this disease and its diagnosis in Slovakia. The study is enriched by comparison of genetic findings from Slovak patients to patients from other countries.

METHODS: Molecular-genetic analysis was performed for patients suspected of SBMA. Data of patients with confirmed diagnosis were statistically evaluated. In addition, the detection rate and the prevalence of the disease for Slovakia were estimated.

RESULTS: In 40 patients with confirmed diagnosis of SBMA, average values were observed at 44.7 CAG repeats and 52.5 years at the time of molecular-genetic diagnosis. The detection rate represents approximately $23 \%$ and an estimated prevalence is of $1: 41,700$.

CONCLUSION: Concerning the population of Slovakia with 5,420,000 inhabitants, we document a relatively large cohort of SBMA patients. This is obvious when comparing similar studies from other countries, while this is the only study representing the Central Europe. Our findings prove that molecular-genetic analyses for the detection of this neuromuscular disorder show high efficiency. This fact underlines the necessity of such testing and may serve as a guide for clinicians from other countries in setting the right diagnosis for these patients (Tab. 1, Fig. 2, Ref. 29). Text in PDF www.elis.sk.

KEY WORDS: androgen receptor, spinal and bulbar muscular atrophy, motor neuron disease, CAG repeats, polyglutamine tract.

\section{Introduction}

Spinal and bulbar muscular atrophy (SBMA) (MIM\# 313200) is a neuromuscular hereditary disorder and belongs to the group of polyglutamine (polyQ) expansion diseases (1). SBMA affects especially lower motor neurons, which are responsible for muscle movements (2). Clinical, pathological and genetic features of this disorder were first described by Kennedy et al in 1968 (3) and thus were originally referred to as Kennedy disease. The molecular

${ }^{1}$ Institute of Medical Biology, Genetics and Clinical Genetics, Comenius University Faculty of Medicine and University Hospital in Bratislava, Department of molecular and biochemical genetics, Bratislava, Slovakia, and ${ }^{2}$ Center for Neuromuscular Diseases, Slovak Medical University and University Hospital in Bratislava, Department of neurology, Ružinov Hospital, Bratislava, Slovakia

Address for correspondence: H.Zelinkova, Institute of Medical Biology, Genetics and Clinical Genetics, Mickiewiczova 13, SK-813 69 Bratislava, Slovakia.

Phone: +421.902621313

Acknowledgements: This work is the result of implementing the project "University Scientific Park of the Comenius University in Bratislava" (ITMS 26240220086), supported by the Research and Development Operational Programme funded by the European Regional Development Fund, and was supported also by the Grant of Comenius University for young researchers, Nr. UK/280/2012 and Nr. UK/344/2013.

We thank RNDr. Ján Luha, CSc., for statistical analysis. cause of SBMA was characterized by La Spada et al in 1991 (4) as an abnormal expansion of CAG repetitions in the first exon of androgen receptor $(A R)$ gene.

$A R$ gene encodes AR protein, which is a member of nuclear receptor family and functions as a transcription factor mediating the androgen actions. Trinucleotide repetition $\mathrm{CAG}$ gives rise to a polyQ tract within N-terminal domain of the protein. SBMA manifestations are supposed to result from the toxic gain in function of the AR with expanded polyQ tract, which is simultaneously dependent on the binding of androgenic ligand (testosterone or dihydrotestosterone) $(5,6)$. Accumulation of ARs containing excessively long polyQ tracts and formation of intranuclear inclusions leads to proteolytic cleavage, subsequent cell apoptosis and finally to motor neuron loss (7).

The number of CAG repeats is highly variable in different individuals as well as in different ethnicities and races (8). Normal distribution of these repeats is set to be 6-35 repeats in general with an average number of 22-23 repetitions in Caucasian population (9). The number of these repetitions in all SBMA patients reaches at least $38(10)$ and the longest expansion reported to date is 68 repeats (11). An inverse relation exists between the number of CAG repeats and transactivation potential of AR. This is the reason why expanded polyQ tract could be associated with reduced AR transcriptional activity and thus also with symptoms resulting from reduced androgen action (12). In SBMA, the number of 
137-141

CAG repeats directly correlates with the severity of the disease and inversely with age at disease onset (13). Practically, patients with higher number of CAG repeats develop the signs and symptoms of SBMA at an earlier age and have them more severe.

Together with amyotrophic lateral sclerosis, SBMA is the most common disorder in the group of motor neuron diseases (14), with prevalence estimated to be one case in 50,000 men (15). As to clinical manifestations, SBMA is observed only in men; women are carriers and are usually asymptomatic. Some heterozygous or homozygous women may sometimes exhibit mild neurological manifestations of the disease (16), such as abnormal electromyograms and presence of occasional cramps and tremor at an advanced age. Muscle cramps, fatigue, and mild CK elevation can be early symptoms in young males (17). Main neurological symptoms start in the fourth decade with insidious slowly progressive weakness and atrophy of the bulbar and proximal limb muscles. Rarely, a rapid progression course is noted. Muscle weakness and wasting in arms and legs slowly get worse in time and result in difficulties with walking and tendency to fall. Tongue atrophy and fasciculations are often noted. Facial and perioral grouped fasciculations are characteristic features of the disease. The neurologic impairment is usually without major bulbar and respiratory dysfunction, and long-term survival is only mildly reduced (18). Patients may also present with mild androgen insensitivity, which can manifest as gynecomastia, hypogonadism, and reduced fertility (19). Also higher-order frontotemporal functions have been found to be particularly vulnerable in SBMA patients (14). An overall treatment to alter the course of the disease is not available and only symptomatic treatment is possible (13). Several clinical studies focused on possible therapies have been carried out, but none of them was effective enough. Further investigations in this field will be needed in the future.

In this paper we describe experiences with molecular-genetic diagnosis of SBMA in Slovakia, which has been performed at our department during past eight years. Few studies focused on SBMA diagnosis in different populations have been published. Therefore we compare our findings with those of other authors and discuss obtained results.

\section{Materials and methods}

\section{Subjects}

Cohort included in our study represents patients suspected of being affected by SBMA. Patients were selected mainly by neurologist on the basis of medical history, neurological, laboratory, and electrophysiological examinations. All individuals in this study provided an informed written consent and, in case of a positive finding, they were offered genetic counselling.

\section{PCR, fragmentation and sequencing analysis}

Genomic DNA was isolated from peripheral blood leukocytes. PCR products for fragmentation and sequencing analyses were amplified using a pair of primers with following sequences: forward: 5'-TCCAGAATCTGTTCCAGAGCGTGC-3' and reverse: 5'-GCTGTGAAGGTTGCTGTTCCTCAT-3', while forward primer was fluorescently end-labeled. The required sequence was amplified using $25 \mathrm{ng}$ of genomic DNA, $0.24 \mu \mathrm{mol} / \mathrm{l}$ of each primer and Master Mix given by the supplier (Thermo Fisher Scientific) in a final volume of $25 \mu \mathrm{l}$. The amplification conditions were as follows: initial denaturing step of $95^{\circ} \mathrm{C}$ for 4 min.; 28 cycles of $95^{\circ} \mathrm{C}$ for 45 $\mathrm{s}, 58^{\circ} \mathrm{C}$ for $45 \mathrm{~s}, 72^{\circ} \mathrm{C}$ for $45 \mathrm{~s}$; followed by a final extension step at $72^{\circ} \mathrm{C}$ for $25 \mathrm{~min}$. Amplified DNA fragments were analysed by agarose gel electrophoresis, using $2 \%$ agarose gel on $100 \mathrm{~V}$ for 60 minutes. The number of CAG repetitions was calculated by sizing PCR products using ABI PRISM Genetic Analyser 310, with comparison to standard size marker TAMRA 500 (Applied Biosystems) and controls with known sizes of CAG repeats, which were confirmed by sequencing. Sequence analysis was performed to confirm the number of CAG repeats in controls or when a threshold number of $\mathrm{CAG}$ repeats had been identified. The sequencing procedure matched standard approaches, while samples were directly sequenced with the ABI PRISM Genetic Analyser 3100 (Applied Biosystems). All sequence data were compared to the published human AR gene sequence (GenBankAccesion No. NG_009014.2) using programs Chromas 2.2 and Vector NTI Advance 11.

\section{Statistical analysis}

Quantitative data for SBMA patients were summarized using descriptive statistics, including mean, median, SD, range, percentages, and $95 \%$ confidence interval of mean. Calculations were performed using the statistical software IBM SPSS Statistics 20.

\section{Results}

We document a cohort of 174 male individuals analysed for SBMA. The diagnosis has been confirmed in 40 of them. We analysed also 17 females for the purpose of determining the carrier state. This was confirmed in 10 women. The number of men in which SBMA has been confirmed represents approximately $23 \%$ of all patients analysed, which means that in about every fourth man with suspected diagnosis the disease was confirmed. The mean value of CAG repeats in patients with SBMA was identified at 44.7 \pm 3 (median $45 \pm 3$ ) and the repeats ranged from 38 to 51 (Fig. 1A). The mean age at the time of setting the diagnosis of SBMA was $52.5 \pm 10$ years (median $53 \pm 10$ ) and included men from 31 to 78 years of age (Fig. 1B). In the cohort of 134 individuals assigned as healthy, we found that the mean number of CAG repeats was $20.9 \pm 4$ (median $21 \pm 4$ ), ranging from 13 to 29 (Fig. 1A). Among the total 174 male samples analysed, we do not register values of CAG repeats in the range of 30-37.

When reviewing the past eight years of molecular-genetic analysis of SBMA at our institute, we observe a gradual increase in the number of individuals required for diagnosis in this period (Fig. 2A). Considering the ratio of individuals with confirmed diagnosis of SBMA to total numbers of analysed individuals during these years, we see that the proportion of confirmed diagnoses per year is statistically significantly variable $(p=0.021)$ (Fig. 2B).

We also tried to estimate the prevalence of SBMA for Slovak population and to compare it with the general prevalence of SBMA. The result was an approximate prevalence of $1: 41,7000$. 


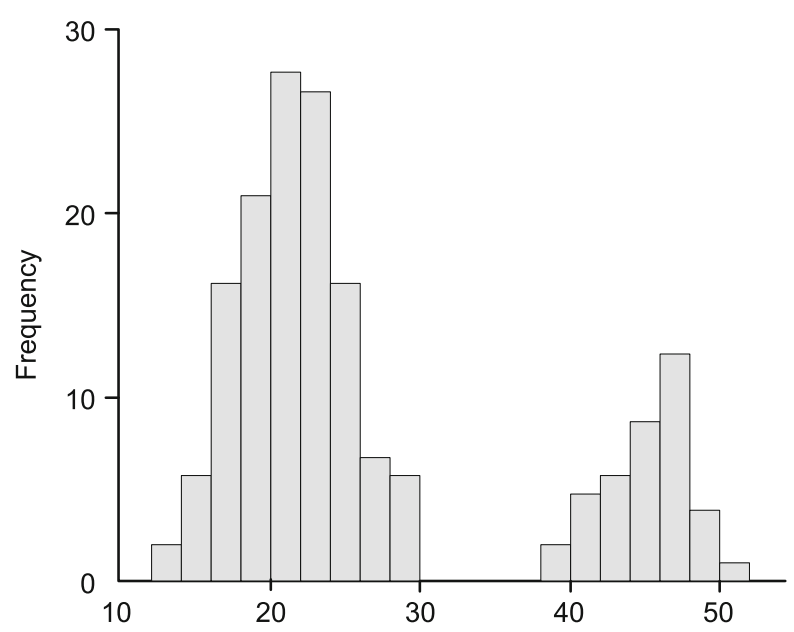

(A)

Number of CAG repeats

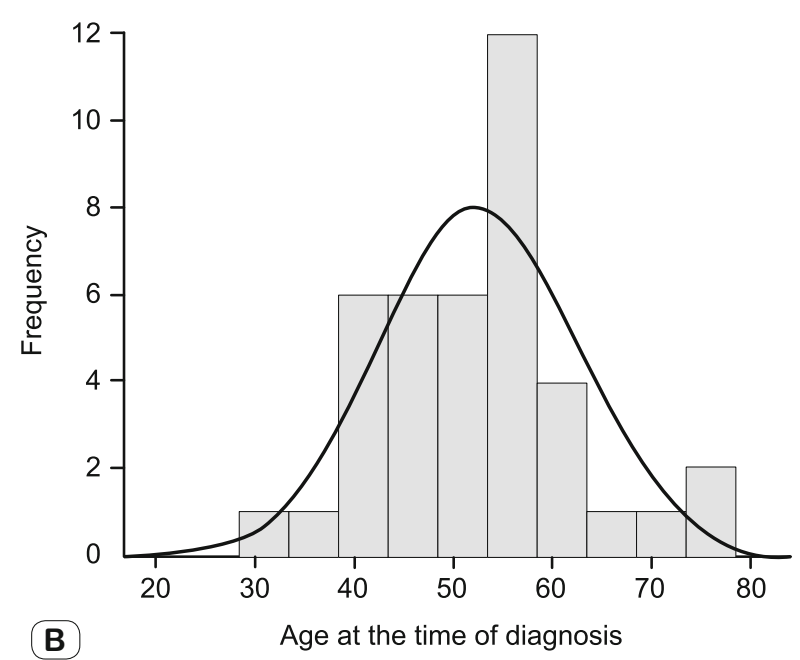

Fig. 1.Genetic correlations. Histogram A illustrates distribution of values of CAG repeats in individuals identified as healthy vs. individuals with SBMA. Histogram B represents frequency of ages of SBMA patients at the time of molecular-genetic diagnosis.
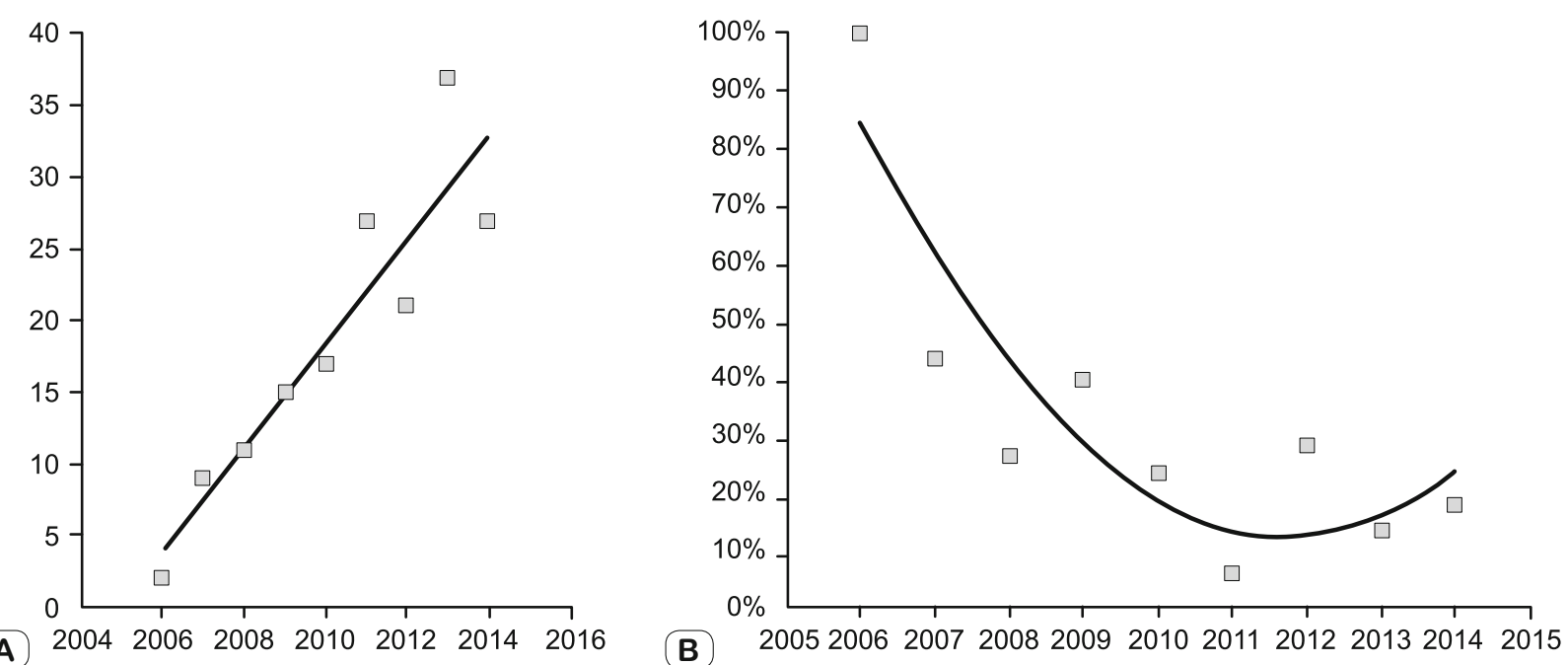

Fig. 2.History of SBMA detection at our institute throughout years 2006-2014. Graph A shows annual number of patients accepted for molecular-genetic analysis of SBMA. We can observe a gradual increase in number of analysed individuals. Graph $B$ represents detection rate of SBMA patients. Portion of individuals with confirmed diagnosis of SBMA is significantly changing, while the course is best characterized by polynomial regression of the whole period.

\section{Discussion}

Molecular basis of SBMA is an excessive expansion of number of CAG repeats in the first exon of the $A R$ gene. Neurological manifestations of SBMA result from motor neuron loss due to toxic gain of function of the altered protein (5). The number of CAG repeats that is considered to cause the disease is set to 38 and more (10).

During the past eight years of the molecular diagnosis of SBMA at our department, we have analysed 191 suspect patients sent from physicians in different parts of Slovakia. The total number consists of 174 male and 17 female samples. The diagnosis of SBMA was confirmed in 40 men. This represents approximately $23 \%$, which means that for about every fourth man, the diagno- sis of SBMA was confirmed. We consider this detection rate of affected men to be very successful and pointing out to both high efficiency of patient selection by physicians and their reasonable indications for molecular-genetic testing.

As the study was performed at a clinical genetic laboratory, we considered mainly molecular-genetic findings. These reveal that the average number of CAG repeats in men with confirmed diagnosis of SBMA was $44.7 \pm 3$, value of median was $45 \pm 3$, whereas the range included values from 38 to 51 repeats. This finding corresponds with data published by other authors, as summarized in Table 1. Although median is of a more informative value, most authors of similar studies report mean values. For this reason, when comparing our findings with other studies, we use both values. 
Tab. 1.Genetic data of SBMA patients from various countries.

\begin{tabular}{|c|c|c|c|c|c|c|}
\hline Year of the study & Country & Case no. & Mean CAG repeat no. & Median CAG repeat no. & Range of CAG repeats & Reference \\
\hline 2000 & Italy & 40 & $44.6 \pm 2.3$ & - & $39-50$ & 20 \\
\hline 2005 & Korea & 18 & $49.8 \pm 2.6$ & - & $46-54$ & 21 \\
\hline 2006 & Japan & 223 & $46.6 \pm 3.5$ & - & $40-57$ & 17 \\
\hline 2008 & Japan & 97 & $47.8 \pm 3.1$ & - & $41-57$ & 22 \\
\hline 2009 & USA & 57 & $46.7 \pm 2.5$ & - & $41-53$ & 23 \\
\hline 2013 & Taiwan & 21 & $47 \pm 3$ & - & $42-53$ & 24 \\
\hline 2014 & UK & 39 & 46.03 & - & $40-53$ & 25 \\
\hline 2015 & Korea & 40 & - & 44 & $39-55$ & 27 \\
\hline Current study & Slovakia & 40 & $44.7 \pm 3$ & $45 \pm 3$ & $38-51$ & - \\
\hline
\end{tabular}

The average age of men with SBMA at the time of moleculargenetic analysis was observed to be $52.5 \pm 10$ years, although we analysed men at age from 31 to 78 . Median was $53 \pm 10$. Clinical symptoms usually show up in the fourth decade of life, but until the patient visits the physician with some disease complaints, it may take a few years, and thus the setting of the diagnosis in patient's fifties is quite relevant. In addition, it has to be taken into consideration that the age at onset and severity of symptoms are in most of cases represented by uncertain data. Another important assumption is to be considered, namely that if the moleculargenetic analysis had been available earlier, the mean age at the time of confirming the definite diagnosis would have also been lower. For comparison, Rhodes et al (23) found that the average age at confirming SBMA diagnosis in USA was 47 years with an average delay of 3.5 years from the first neurological examination to molecular-genetic diagnosis. A proportion of $32 \%$ of all their patients were misdiagnosed before setting the final SBMA diagnosis. Also Fratta et al (25) indicate that 1 out of 3 patients in their study received an alternative diagnosis before the right one. $\mathrm{Ni}$ et al (26) noticed the mean age at genetic diagnosis of their patients was $51.0 \pm 10.1$ (range 25-74) and patients of Song et al (27) had the median age at diagnosis 52.5 (range 34-74). These findings correspond with ours.

When evaluating 134 individuals in which the diagnosis of SBMA has not been confirmed, we found that the average number of their CAG repeats was $20.9 \pm 4$, ranging from 13 to 29 repeats. In this case, the median value was $21 \pm 4$. We did not observe values of CAG repeats in the interval of numbers 30-37, which indicates that the hiatus between physiological and pathological numbers of repeats is unequivocal. The genetic mechanism of this observation is not clear, but most likely it results from the phenomenon of anticipation, which is common among polyQ diseases.

After reviewing the history of molecular-genetic analysis of SBMA at our institute during eight years, we see that the number of samples accepted and analysed was gradually increasing, while the highest number of patients diagnosed so far took place in 2013. The detection rate resulting from the number of confirmed SBMA patients to all individuals analysed per year is quite variable. It may seem that in the period from 2006 to 2010, the captivity was higher than in years 2011-2014. However we consider the situation concerning the detection rate of positive patients to be quite stable mainly in the last five years with the mean of $18.3 \%$ during this period.
As the prevalence of SBMA is mostly referred as $1: 50,000$, we tried to set the prevalence for the Slovak Republic. We considered the age interval covering statistically significant $95 \%$ based on information about our SBMA patients. The average number of all men from this interval in each observed year was calculated and was divided by the number of all positive SBMA patients detected during this period. We found out that our results were not consistent with the general information, as our calculation has lead us to prevalence as high as $1: 41,700$. In addition, the given general prevalence of SBMA of $1: 50,000$ should lead us to 53 affected men covering all age groups in Slovakia (according to data provided by (28)). Our institute with a nationwide scope has already collected 40 individuals at an age range of 31-78. This fact supports our assumption, that the prevalence of SBMA in Slovakia would be higher than estimated generally. Some other sources, as e.g. Orphanet, refer that the prevalence of SBMA is 1 in 30,000 male births (29) and some authors mention also prevalence of 1 : 40,000 (5). We have to take into account also the fact that SBMA is considered to be an underdiagnosed disease, and in reality, many SBMA patients may be received an incorrect diagnosis. These false diagnoses may result from several factors, including the lack of experience or knowledge of physicians, especially neurologists and geneticists, and similarities in manifestations with other degenerative motor neuron diseases. Moreover, it may be sometimes problematic to recognize the X-linked mode of inheritance and the disease may be overlooked since some patients exhibit only mild symptoms during slow and gradual course of SBMA.

\section{Conclusion}

In this study, we report experience with molecular-genetic diagnosis of SBMA in Slovakia, while this is the first report of its kind in the country from Central Europe. Given the population number of Slovakia in comparison with other countries, we document a relatively large cohort of SBMA patients. SBMA is one of the most common motor neuron disorders and confirmatory genetic testing is particularly important for rapid differentiation of SBMA from other neurodegenerative disorders with significantly worse prognosis. Our findings indicate that these analyses were very effectively used, as the SBMA detection rate was approximately $23 \%$. The correlation of the number of CAG repeats to clinical findings such as age at onset and also severity of symptoms of the disease should be subject to further clinical study. 


\section{Learning points}

- We report the status of molecular-genetic diagnosis of spinal and bulbar muscular atrophy in Slovakia.

- In 40 patients with confirmed diagnosis, the average $\mathrm{CAG}$ repeat size was 44.7 .

- The age at confirming the diagnosis was 52.5 years on average.

- An estimated prevalence resulting from our findings was of 1 : 41,700 .

- We note approximately $23 \%$ detection rate in patients with the disease.

\section{References}

1. Cortes CJ, Ling SC, Guo LT et al. Muscle expression of mutant androgen receptor accounts for systemic and motor neuron disease phenotypes in spinal and bulbar muscular atrophy. Neuron 2014; 82 (2): 295-307.

2. Scaramuzzino C, Casci I, Parodi S et al. Protein arginine methyltransferase 6 enhances polyglutamine-expanded androgen receptor function and toxicity in spinal and bulbar muscular atrophy. Neuron 2015; 85 (1): $88-100$.

3. Kennedy WR, Alter M, Sung JH. Progressive proximal spinal and bulbar muscular atrophy of late onset. A sex-linked recessive trait. Neurology 1968; 18 (7): 671-680.

4. La Spada AR, Wilson EM, Lubahn DB, Harding A, Fischbeck KH. Androgen receptor gene mutations in X-linked spinal and bulbar muscular atrophy. Nature 1991; 352 (6330): 77-79.

5. Grunseich C, Rinaldi C, Fischbeck KH. Spinal and bulbar muscular atrophy: pathogenesis and clinical management. Oral Dis 2014; 20 (1): 6-9.

6. Giorgetti E, Rusmini P, Crippa V et al. Synergic prodegradative activity of Bicalutamide and trehalose on the mutant androgen receptor responsible for spinal and bulbar muscular atrophy. Hum Mol Genet 2015; 24 (1): 64-75.

7. Heine EM, Berger TR, Pluciennik A, Orr CR, Zboray L, Merry DE. Proteasome-mediated Proteolysis of the Polyglutamine-expanded Androgen Receptor Is a Late Event in Spinal and Bulbar Muscular Atrophy (SBMA) Pathogenesis. J Biol Chem 2015; 290 (20): 12572-12584.

8. Ackerman CM, Lowe LP, Lee $\mathbf{H}$ et al. Ethnic variation in allele distribution of the androgen receptor (AR) (CAG)n repeat. J Androl 2012; 33 (2): 210-205.

9. Nenonen HA, Giwercman A, Hallengren E, Giwercman YL. Nonlinear association between androgen receptor CAG repeat length and risk of male subfertility--a meta-analysis. Int J Androl 2011; 34 (4): 327-332.

10. Rocchi A, Pennuto M. New routes to therapy for spinal and bulbar muscular atrophy. J Mol Neurosci 2013; 50 (3): 514-523.

11. Grunseich C, Kats IR, Bott LC et al. Early onset and novel features in a spinal and bulbar muscular atrophy patient with a 68 CAG repeat. Neuromuscul Disord 2014; 24 (11): 978-981.

12. Gottlieb B, Beitel LK, Nadarajah A, Paliouras M, Trifiro M. The androgen receptor gene mutations database: 2012 update. Hum Mutat 2012; 33 (5): 887-894.
13. Grunseich C, Zukosky K, Kats IR et al. Stem cell-derived motor neurons from spinal and bulbar muscular atrophy patients. Neurobiol Dis 2014; 70: 12-20.

14. Di Rosa E, Sorarù G, Kleinbub JR et al. Theory of mind, empathy and neuropsychological functioning in X-linked Spinal and Bulbar Muscular Atrophy: a controlled study of 20 patients. J Neurol 2015; 262 (2): 394-401.

15. Beitel LK, Alvarado C, Mokhtar S, Paliouras M, Trifiro M. Mechanisms mediating spinal and bulbar muscular atrophy: investigations into polyglutamine-expanded androgen receptor function and dysfunction. Front Neurol 2013; 4: 53.

16. Parodi S, Pennuto M. Neurotoxic effects of androgens in spinal and bulbar muscular atrophy. Front Neuroendocrinol 2011; 32 (4): 416-425.

17. Atsuta $\mathbf{N}$, Watanabe $\mathbf{H}$, Ito $\mathbf{M}$ et al. Natural history of spinal and bulbar muscular atrophy (SBMA): a study of 223 Japanese patients. Brain 2006; 129 (Pt 6): 1446-1455.

18. Chahin N, Klein C, Mandrekar J, Sorensen E. Natural history of spinal-bulbar muscular atrophy. Neurology 2008; 70 (21): 1967-1971.

19. Dossena M, Bedini G, Rusmini P et al. Human adipose-derived mesenchymal stem cells as a new model of spinal and bulbar muscular atrophy. PLoS One 2014; 9 (11): e112746.

20. Mariotti C, Castellotti B, Pareyson D et al. Phenotypic manifestations associated with CAG-repeat expansion in the androgen receptor gene in male patients and heterozygous females: a clinical and molecular study of 30 families. Neuromuscul Disord 2000; 10 (6): 391-397.

21. Lee JH, Shin JH, Park KP et al. Phenotypic variability in Kennedy's disease: implication of the early diagnostic features. Acta Neurol Scand 2005; 112 (1): 57-63.

22. Suzuki K, Katsuno M, Banno $\mathbf{H}$ et al. CAG repeat size correlates to electrophysiological motor and sensory phenotypes in SBMA. Brain 2008; 131 (Pt 1): 229-239.

23. Rhodes LE, Freeman BK, Auh $\mathrm{S}$ et al. Clinical features of spinal and bulbar muscular atrophy. Brain 2009; 132 (Pt 12): 3242-3251.

24. Fu SC, Kuo HC, Chu CC et al. Long-term follow-up of spinal and bulbar muscular atrophy in Taiwan. J Formos Med Assoc 2013; 112 (6): $326-331$.

25. Fratta P, Nirmalananthan N, Masset $\mathbf{L}$ et al. Correlation of clinical and molecular features in spinal bulbar muscular atrophy. Neurology 2014; 82 (23): 2077-2084.

26. Ni W, Chen S, Qiao K, Wang N, Wu ZY. Genotype-phenotype correlation in Chinese patients with spinal and bulbar muscular atrophy. PLoS One 2015; 10 (3): e0122279.

27. Song JS, Kim KA, Min JH, Ki CS, Kim JW, Sung DH, Kim BJ. Clinical Characteristics and Genotype-Phenotype Correlation of Korean Patients with Spinal and Bulbar Muscular Atrophy. Yonsei Med J 2015; 56 (4): 993-997.

28. www.statistics.sk.

29. http://www.orpha.net/consor/cgi-bin/index.php?lng=EN.

Received June 9, 2015. Accepted October 27, 2015. 